OPEN ACCESS

Edited by:

Yu Ru Kou,

National Yang-Ming University, Taiwan

Reviewed by:

Michaela Kollisch-Singule, Arkansas Children's Hospital,

United States

Zhanqi Zhao,

Furtwangen University, Germany

${ }^{*}$ Correspondence:

Gaetano Perchiazzi

gaetano.perchiazzi@surgsci.uu.se

Specialty section:

This article was submitted to Respiratory Physiology, a section of the journal

Frontiers in Physiology

Received: 17 October 2020 Accepted: 22 December 2020 Published: 09 February 2021

Citation:

Pellegrini $M$, Hedenstierna $G$, Larsson AS and Perchiazzi G (2021) Inspiratory Efforts, Positive End-Expiratory Pressure, and External Resistances Influence Intraparenchymal Gas Redistribution in Mechanically Ventilated Injured Lungs. Front. Physiol. 11:618640. doi: 10.3389/fphys.2020.618640

\section{Inspiratory Efforts, Positive End-Expiratory Pressure, and External Resistances Influence Intraparenchymal Gas Redistribution in Mechanically Ventilated Injured Lungs}

\author{
Mariangela Pellegrini1,2, Göran Hedenstierna ${ }^{3}$, Anders Sune Larsson ${ }^{1}$ and \\ Gaetano Perchiazzi1,2*
}

\begin{abstract}
${ }^{1}$ Hedenstierna Laboratory, Department of Surgical Sciences, Uppsala University, Uppsala, Sweden, ${ }^{2}$ Intensive Care Unit, Department of Anesthesia, Operation and Intensive Care, Uppsala University Hospital, Uppsala, Sweden, ${ }^{3}$ Hedenstierna Laboratory, Department of Medical Sciences, Uppsala University, Uppsala, Sweden
\end{abstract}

Background: Potentially harmful lung overstretch can follow intraparenchymal gas redistribution during mechanical ventilation. We hypothesized that inspiratory efforts characterizing spontaneous breathing, positive end-expiratory pressure (PEEP), and high inspiratory resistances influence inspiratory intraparenchymal gas redistribution.

Methods: This was an experimental study conducted on a swine model of mild acute respiratory distress syndrome. Dynamic computed tomography and respiratory mechanics were simultaneously acquired at different PEEP levels and external resistances, during both spontaneous breathing and controlled mechanical ventilation. Images were collected at two cranial-caudal levels. Delta-volume images ( $\Delta$ VOLs) were obtained subtracting pairs of consecutive inspiratory images. The first three $\Delta$ VOLs, acquired for each analyzed breath, were used for the analysis of inspiratory pendelluft defined as intraparenchymal gas redistribution before the start of inspiratory flow at the airway opening. The following $\Delta \mathrm{VOLs}$ were used for the analysis of gas redistribution during ongoing inspiratory flow at the airway opening.

Results: During the first flow-independent phase of inspiration, the pendelluft of gas was observed only during spontaneous breathing and along the cranial-to-caudal and nondependent-to-dependent directions. The pendelluft was reduced by high PEEP $\left(p<0.04\right.$ comparing PEEP 15 and PEEP $\mathrm{O} \mathrm{cm} \mathrm{H}_{2} \mathrm{O}$ ) and low external resistances ( $p<0.04$ comparing high and low external resistance). During the flow-dependent phase of inspiration, two patterns were identified: (1) gas displacing characterized by large gas redistribution areas; (2) gas scattering characterized by small, numerous areas of gas redistribution. Gas displacing was observed at low PEEP, high external resistances, and it characterized controlled mechanical ventilation $(p<0.01$, comparing high and low PEEP during controlled mechanical ventilation). 
Conclusions: Low PEEP and high external resistances favored inspiratory pendelluft. During the flow-dependent phase of the inspiration, controlled mechanical ventilation and low PEEP and high external resistances favored larger phenomena of intraparenchymal gas redistribution (gas displacing) endangering lung stability.

Keywords: assisted mechanical ventilation, lung heterogeneity, mild acute respiratory distress syndrome, CT imaging, self-induced lung injury

\section{INTRODUCTION}

The intraparenchymal gas redistribution occurring in absence of flow at the airway opening has been defined for the first time in 1956 and named pendelluft (Otis et al., 1956). The magnitude of this phenomenon is closely related to the heterogeneity of lung injury and to the ventilatory settings. The heterogeneity in regional compliance and resistance, characterizing injured lungs (Harada et al., 1983; Sala et al., 2002; Perlman et al., 2010; Vyshedskiy and Murphy, 2012; Yoshida et al., 2016b), leads to unstable acinar structures and transient pressure gradients among adjacent lung regions, consequently causing gas redistribution within the lung parenchyma (Yoshida et al., 2016a; Brochard et al., 2017). Moreover, suboptimal ventilatory settings and strong inspiratory efforts further enhance heterogeneity between contiguous regions and promote lung instability (Otis et al., 1956; Mead et al., 1970; Ultman et al., 1988; Greenblatt et al., 2014). Recent studies show harmful inspiratory pendelluft following high inspiratory efforts when spontaneous breathing is allowed in mechanically ventilated subjects with acute respiratory distress syndrome (ARDS) (Yoshida et al., 2013; Morais et al., 2018). Consequently, the scientific community debates whether and to which extent to allow spontaneous breathing in mechanically ventilated subjects affected by ARDS (Papazian et al., 2010; Haren et al., 2019; Moss et al., 2019; Schepens et al., 2019).

To investigate all possible factors causing intraparenchymal gas redistribution is of crucial importance for understanding the mechanisms leading to ventilator-induced lung injury. In the current experimental study, we investigated if spontaneous inspiratory efforts, positive endexpiratory pressure (PEEP), and external resistances influenced onset and magnitude of intraparenchymal gas redistribution:

(a) during the first isovolumetric, flow-independent phase of the inspiration defined as pendelluft (Yoshida et al., 2013);

(b) during ongoing inspiratory flow at the airway opening.

\section{MATERIALS AND METHODS}

The current study was approved by the regional animal ethics committee (No. C 46_14) and performed at the Hedenstierna Laboratory (Uppsala University, Sweden). Detailed information about the methods has been provided in the online data supplement.

\section{Experimental Setting and Protocol}

Six anesthetized, mechanically ventilated pigs $(29.9 \pm 2.6 \mathrm{~kg})$ underwent repeated lung lavages $(30 \mathrm{ml} / \mathrm{kg}$ of warmed isotonic saline at $37^{\circ} \mathrm{C}$ ) to create a surfactant-deficient mild ARDS injury $\left(\mathrm{PO}_{2} / \mathrm{F}_{\mathrm{I}} \mathrm{O}_{2}\right.$ of $250 \mathrm{mmHg}$ at a PEEP of $\left.5 \mathrm{~cm} \mathrm{H} \mathrm{H}_{2} \mathrm{O}\right)$. The animals were placed in supine position. Spontaneous breathing (continuous positive airway pressure) and controlled mechanical ventilation in fully paralyzed conditions (pressure-controlled ventilation) were applied at comparable tidal volumes and respiratory rates. Spontaneous breathing was performed at two different external airway resistances: high (SB-HighR) and low (SB-LowR) resistance. SB-LowR was achieved by applying an endotracheal tube with an internal diameter of $9 \mathrm{~mm}$. SB-HighR was achieved by applying an endotracheal tube with an internal diameter of $6 \mathrm{~mm}$. During both spontaneous breathing and controlled mechanical ventilation, six PEEP levels $(15,12,9$, 6,3 , and $0 \mathrm{~cm} \mathrm{H}_{2} \mathrm{O}$ ) were applied. A steady state was always restored between two consecutive tested ventilatory conditions. Respiratory mechanics, electrical activity of the diaphragm (EAdi), and dynamic CT scans were simultaneously recorded. Dynamic CT images were recorded 1 (L1) and $4 \mathrm{~cm}$ (L4) above the diaphragmatic dome, with an acquisition rate of 20 images per second.

\section{Data Analysis}

The volume tracings derived from simultaneously acquired CT scans allowed the synchronization between CT images and spirometric data, as done in Pellegrini et al. (2017), thus allowing breath-by-breath analysis of tracings and images in-phase. All acquired inspiratory CT images were further processed to obtain delta-volume images ( $\Delta$ VOLs) between consecutive CT images. Each delta volume was characterized by a 0.05 -s time frame.

\section{Intraparenchymal Gas Redistribution Before the Onset of Inspiratory Flow (Pendelluft)}

The inspiratory pendelluft was defined as intraparenchymal gas redistribution when the inspiratory effort has not yet induced an inspiratory flow at the airway opening (Otis et al., 1956; Yoshida et al., 2013). During this phase, the internal redistribution of gas is possible. The time related to auto-PEEP was calculated to estimate the time of isovolumetric inspiration (see Figure 1). During spontaneous breathing, dynamic autoPEEP was calculated as the difference in Pes between the point of zero flow at end expiration and the onset of the subsequent inspiratory flow. During controlled mechanical ventilation, autoPEEP was estimated by calculating the difference in airway 


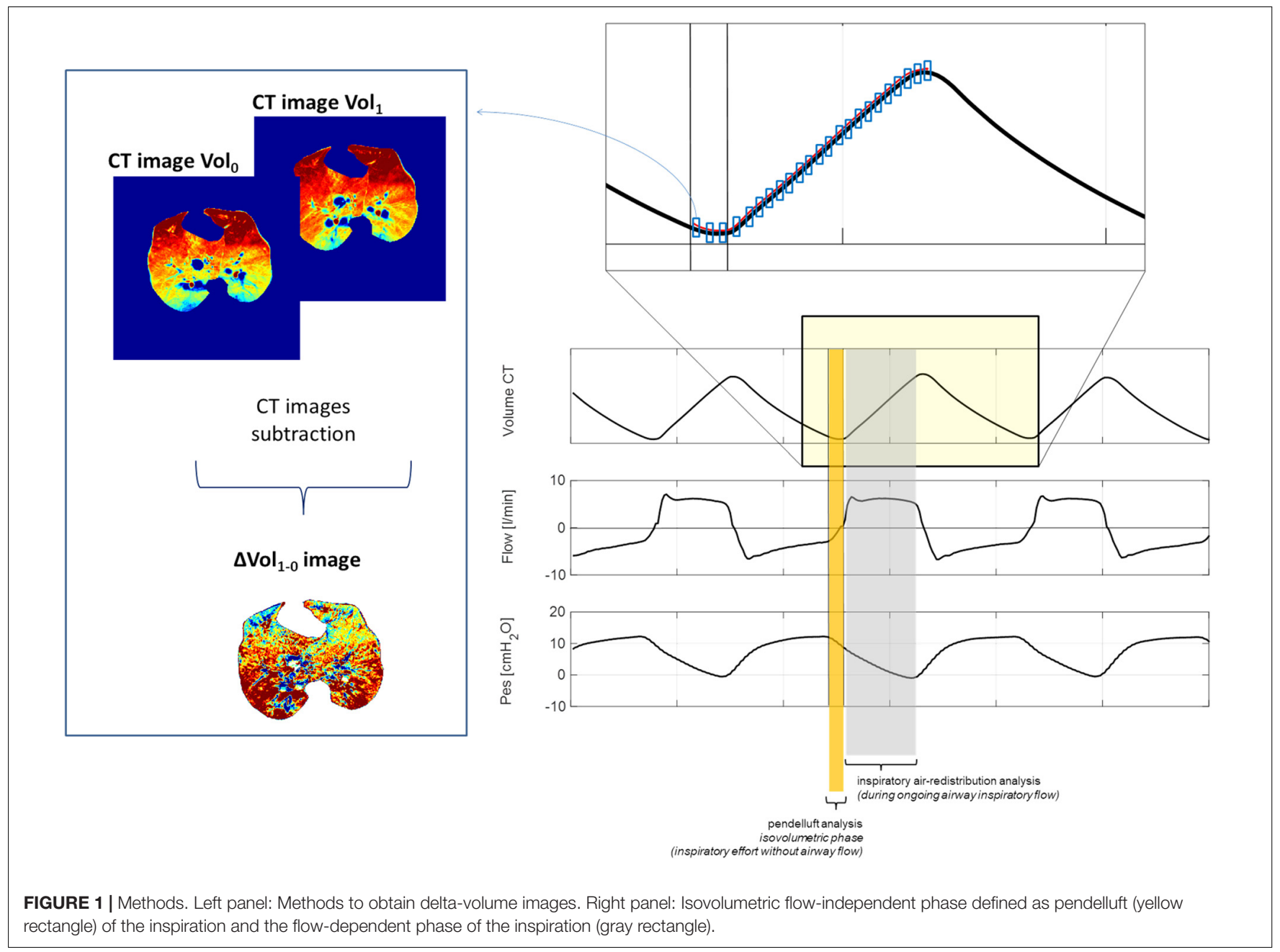

pressure in the time frame between end expiration and the onset of inspiratory flow (Rossi et al., 1995). The number of CT images at the beginning of the inspiration to be included in the pendelluft analysis was determined based on the longest recorded time necessary to overcome auto-PEEP in the entire group of studied animals. To explore the inspiratory pendelluft, four CT images (and the corresponding three $\triangle$ VOLs, covering the first $0.15 \mathrm{~s}$ of each inspiration) were selected at the beginning of each inspiration (see Figure 1). Each $\triangle \mathrm{VOL}$ was then divided into four equally sized regions of interest (ROI1, ROI2, ROI3, and ROI4), with ROI1 being the most nondependent and ROI4 the most dependent one. For each ROI, gas flow was computed and normalized to the extension of the corresponding ROI: ( $\mathrm{ml}$ of gas $/ 0.05 \mathrm{~s} / \mathrm{mm}^{3}$ of ROI extension). Combining four gravitational ROIs and two cranial-caudal levels, a three-dimensional assessment of intraparenchymal gas redistribution was possible.

\section{Intraparenchymal Gas Redistribution During Ongoing Inspiratory Flow}

To reveal patterns of gas redistribution during ongoing inspiratory flow at the airway opening, the quadtree decomposition algorithm was applied to the $\Delta$ VOLs (see Figure 1). The quadtree decomposition is a standard algorithm in image processing $(18,19)$ that iteratively divides the image into four equal squares until a predetermined criterion of homogeneity is satisfied. If the criterion is not satisfied, the analyzed image portion is further divided into four squares. The criterion of homogeneity was defined as a difference in flow between contiguous areas equal to or lower than $10 \%$ (corresponding to a flow difference equal to or lower than $\left.2 \times 10^{-5} \mathrm{ml} / 0.05 \mathrm{~s}\right)$. The mean area of all squares (AreaSq) obtained by the quadtree decomposition algorithm and expressed in $\mathrm{cm}^{2}$ was used to define two patterns of intraparenchymal gas redistribution. For mean AreaSq equal to or lower than the arbitrary threshold of $2 \mathrm{~cm}^{2}$, the gas redistribution pattern was defined as gas scattering; for mean AreaSq higher than $2 \mathrm{~cm}^{2}$, the pattern of gas redistribution was defined as gas displacing.

\section{Statistics}

Normal distribution was confirmed by the one-sample Kolmogorov-Smirnov test $(\alpha=0.05)$. The analysis of variance was used to test statistical differences. Bonferroni's correction 
TABLE 1 | Respiratory mechanics for each studied ventilatory modalities and PEEP level: (A) spontaneous breathing at low external airway resistance; (B) spontaneous breathing at high external airway resistance; $(C)$ controlled mechanical ventilation.

\begin{tabular}{|c|c|c|c|c|c|c|c|}
\hline \multicolumn{8}{|c|}{ Spontaneous breathing low resistance } \\
\hline PEEP & $\begin{array}{l}\text { Tidal volume } \\
\text { (mL) }\end{array}$ & $\begin{array}{l}\text { Unfiltered EAdi } \\
\text { peak (uV) }\end{array}$ & $\begin{array}{l}\text { Neuroventilatory } \\
\text { efficiency (ml/uV) }\end{array}$ & $\begin{array}{c}\Delta \text { Pes } \\
\left(\mathrm{cm} \mathrm{H} \mathrm{H}_{2} \mathrm{O}\right)\end{array}$ & $\begin{array}{l}\text { Auto-PEEP } \\
\left(\mathrm{cm} \mathrm{H} \mathrm{H}_{2} \mathrm{O}\right)\end{array}$ & $\begin{array}{c}\text { Time of } \\
\text { no-flow (s) }\end{array}$ & Ptp $\left(\mathrm{cm} \mathrm{H} \mathrm{H}_{2} \mathrm{O}\right)$ \\
\hline 15 & $225 \pm 7$ & $17.2 \pm 1.8^{\dagger}$ & $13.1 \pm 1.5$ & $-5.06 \pm 0.48^{\star}$ & $0.01 \pm 0.01$ & $0.00 \pm 0.01$ & $20.06 \pm 0.48^{\dagger}$ \\
\hline 12 & $220 \pm 7$ & $13.8 \pm 1.9$ & $16.0 \pm 1.4^{*}$ & $-5.21 \pm 0.27^{\star}$ & $0.25 \pm 0.14$ & $0.02 \pm 0.01$ & $17.21 \pm 0.27^{\dagger}$ \\
\hline 9 & $219 \pm 8$ & $14.1 \pm 1.8$ & $15.6 \pm 1.4^{*}$ & $-6.94 \pm 0.89$ & $0.25 \pm 0.12$ & $0.02 \pm 0.01$ & $15.94 \pm 0.89^{\dagger}$ \\
\hline 6 & $211 \pm 9$ & $13.5 \pm 1.6$ & $15.6 \pm 1.4^{*}$ & $-7.48 \pm 0.83$ & $0.56 \pm 0.19^{\star}$ & $0.03 \pm 0.01$ & $13.48 \pm 0.83^{\dagger}$ \\
\hline 3 & $180 \pm 9^{*}$ & $14.5 \pm 1.0$ & $12.4 \pm 1.0$ & $-8.29 \pm 0.75$ & $1.02 \pm 0.17^{\star}$ & $0.06 \pm 0.01^{*}$ & $11.29 \pm 0.75^{\dagger}$ \\
\hline 0 & $167 \pm 9^{\star}$ & $14.0 \pm 0.7$ & $12.0 \pm 0.9$ & $-7.91 \pm 0.97$ & $1.62 \pm 0.57^{\star}$ & $0.09 \pm 0.01^{\star}$ & $7.91 \pm 0.97^{\dagger}$ \\
\hline \multicolumn{8}{|c|}{ Spontaneous breathing high resistance } \\
\hline PEEP & $\begin{array}{l}\text { Tidal volume } \\
\text { (ml) }\end{array}$ & $\begin{array}{l}\text { Unfiltered EAdi } \\
\text { peak (uV) }\end{array}$ & $\begin{array}{l}\text { Neuroventilatory } \\
\text { efficiency (ml/uV) }\end{array}$ & $\begin{array}{c}\Delta \text { Pes }(\mathrm{cm} \\
\left.\mathrm{H}_{2} \mathrm{O}\right)\end{array}$ & $\begin{array}{l}\text { Auto-PEEP } \\
\left(\mathrm{cm} \mathrm{H} \mathrm{H}_{2} \mathrm{O}\right)\end{array}$ & $\begin{array}{c}\text { Time of } \\
\text { no-flow (s) }\end{array}$ & Ptp $\left(\mathrm{cm} \mathrm{H} \mathrm{H}_{2} \mathrm{O}\right)$ \\
\hline 15 & $214 \pm 4$ & $16.6 \pm 1.9$ & $12.9 \pm 1.1^{*}$ & $-6.98 \pm 1.29^{\dagger}$ & $0.47 \pm 0.12$ & $0.04 \pm 0.01$ & $21.98 \pm 0.48^{*}$ \\
\hline 12 & $215 \pm 9$ & $15.8 \pm 2.1$ & $13.6 \pm 0.9^{*}$ & $-8.28 \pm 1.88$ & $0.84 \pm 0.12$ & $0.05 \pm 0.01$ & $20.28 \pm 0.27^{\star}$ \\
\hline 9 & $215 \pm 5$ & $15.4 \pm 1.6$ & $13.9 \pm 0.9^{*}$ & $-8.93 \pm 1.68$ & $0.81 \pm 0.14$ & $0.06 \pm 0.01$ & $17.93 \pm 0.89^{\dagger}$ \\
\hline 6 & $207 \pm 9$ & $14.3 \pm 1.5$ & $14.5 \pm 1.3^{*}$ & $-8.74 \pm 1.02$ & $1.48 \pm 0.28^{\star}$ & $0.06 \pm 0.01$ & $14.74 \pm 0.83$ \\
\hline 3 & $197 \pm 7$ & $16.6 \pm 1.3$ & $11.8 \pm 1.0$ & $-10.59 \pm 1.94$ & $1.93 \pm 0.36^{\star}$ & $0.10 \pm 0.01$ & $13.59 \pm 0.75$ \\
\hline 0 & $172 \pm 4^{\dagger}$ & $17.8 \pm 0.6^{\star}$ & $9.7 \pm 0.9^{\dagger}$ & $-11.20 \pm 2.50$ & $2.02 \pm 0.29^{\star}$ & $0.13 \pm 0.01$ & $11.20 \pm 0.97^{\dagger}$ \\
\hline \multicolumn{8}{|c|}{ Mechanical ventilation } \\
\hline PEEP & $\begin{array}{l}\text { Tidal volume } \\
\text { (ml) }\end{array}$ & EAdi peak (uV) & $\Delta \mathrm{Pes}\left(\mathrm{cm} \mathrm{H} \mathrm{H}_{2} \mathrm{O}\right)$ & $\begin{array}{l}\text { Auto-PEEP } \\
\left.\text { (cm } \mathrm{H}_{2} \mathrm{O}\right)\end{array}$ & $\begin{array}{c}\text { Time of } \\
\text { no-flow (s) }\end{array}$ & $\begin{array}{c}\text { Pplat } \\
\left.\text { (cm H } \mathrm{H}_{2} \mathrm{O}\right)\end{array}$ & Ptp $\left(\mathrm{cm} \mathrm{H} \mathrm{H}_{2} \mathrm{O}\right)$ \\
\hline 15 & 250 & $0.6 \pm 0.1$ & $0.89 \pm 0.42$ & $0.10 \pm 0.02$ & $0.01 \pm 0.01$ & $21.42 \pm 0.89^{\dagger}$ & $20.53 \pm 0.78^{\dagger}$ \\
\hline 12 & 250 & $0.6 \pm 0.0$ & $0.95 \pm 0.39$ & $0.17 \pm 0.03$ & $0.02 \pm 0.00$ & $18.22 \pm 0.94^{\dagger}$ & $17.27 \pm 0.85^{\dagger}$ \\
\hline 9 & 250 & $0.7 \pm 0.0$ & $1.02 \pm 0.50$ & $0.17 \pm 0.03$ & $0.02 \pm 0.00$ & $15.01 \pm 1.00^{\dagger}$ & $13.99 \pm 0.86^{\dagger}$ \\
\hline 6 & 200 & $0.6 \pm 0.0$ & $0.74 \pm 0.37$ & $0.22 \pm 0.03$ & $0.03 \pm 0.00$ & $12.31 \pm 1.01^{\dagger}$ & $11.57 \pm 0.94^{\dagger}$ \\
\hline 3 & 200 & $0.7 \pm 0.1$ & $0.71 \pm 0.29$ & $0.22 \pm 0.03$ & $0.03 \pm 0.00$ & $9.32 \pm 0.97^{\dagger}$ & $8.61 \pm 0.93^{\dagger}$ \\
\hline 0 & 200 & $0.6 \pm 0.1$ & $0.74 \pm 0.28$ & $0.36 \pm 0.03^{\star}$ & $0.03 \pm 0.00$ & $6.48 \pm 0.97^{\dagger}$ & $5.74 \pm 0.93^{\dagger}$ \\
\hline
\end{tabular}

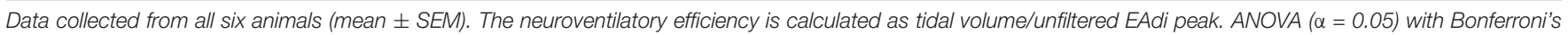

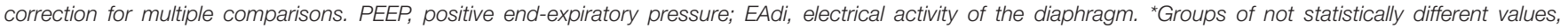
statistically different from others values of the same variable. ${ }^{\dagger}$ Values that are statistically different compared to other values of the same variable.

for multiple comparisons was applied when needed. Descriptive statistics were reported using mean $( \pm$ SEM).

\section{RESULTS}

There were no differences in transpulmonary pressure between spontaneous breathing and controlled mechanical ventilation (see Table 1). This finding made the two tested ventilatory modes comparable in the following phases of the study.

\section{Intraparenchymal Gas Redistribution Before the Onset of Inspiratory Flow (Pendelluft)}

During controlled mechanical ventilation, auto-PEEP was always very low (0.1-0.4 $\mathrm{cm} \mathrm{H}_{2} \mathrm{O}$ ), independently from the PEEP level. During spontaneous breathing, the longest estimated no-flow time at the airway opening at the beginning of inspiration, corresponding to the highest recorded auto-PEEP $\left(2 \mathrm{~cm} \mathrm{H}_{2} \mathrm{O}\right)$, was 0.13 s (see Tables 1, 2 and Supplementary Tables 13). Consequently, considering a time frame of $0.05 \mathrm{~s}$ for each $\triangle$ VOL, the first three $\Delta$ VOLs (at $0.05,0.10$, and $0.15 \mathrm{~s}$, respectively, named $\Delta \mathrm{VOL}_{0.05}, \Delta \mathrm{VOL}_{0.10}$, and $\Delta \mathrm{VOL}_{0.15}$ ) of each analyzed inspiration were selected for the analysis of pendelluft (see Figures 1-3).

During spontaneous breathing, for PEEP levels equal to or higher than $9 \mathrm{~cm} \mathrm{H}_{2} \mathrm{O}$, all ROIs were characterized by a minimal, nonsignificant change in regional volumes (with a regional gas flow $<1.42 \mathrm{E}-06 \mathrm{ml} / \mathrm{mm}^{3}$ and nonstatistical differences between PEEP 15 and PEEP $9 \mathrm{~cm} \mathrm{H} \mathrm{H}_{2} \mathrm{O}$ in ROI1 and ROI2, $\triangle \mathrm{VOL}_{0.05}$ at SB-LowR) (see Figure 2 and Supplementary Figure 1). This was true for both SB-LowR and SB-HighR (with a regional gas flow $<1.29 \mathrm{E}-06 \mathrm{ml} / \mathrm{mm}^{3}$ and nonstatistical differences between PEEP 15 and PEEP $9 \mathrm{~cm} \mathrm{H}_{2} \mathrm{O}$ in ROI1 and ROI2, $\triangle \mathrm{VOL}_{0.05}$ at SB-HighR).

During spontaneous breathing at PEEP levels lower than $9 \mathrm{~cm} \mathrm{H}_{2} \mathrm{O}$, a nondependent cranial (ROI1 and ROI2 at L4) deflation (during $\Delta \mathrm{VOL}_{0.05}$ and $\Delta \mathrm{VOL}_{0.10}$ ) was quasisimultaneous with a dependent caudal (ROI3 and ROI4 at 
TABLE 2 | Auto-PEEP and time of no-flow at the beginning of the inspiration.

\begin{tabular}{|c|c|c|c|c|c|c|}
\hline & \multicolumn{2}{|c|}{ Spontaneous breathing low resistance } & \multicolumn{2}{|c|}{ Spontaneous breathing high resistance } & \multicolumn{2}{|c|}{ Mechanical ventilation } \\
\hline 15 & $0.01 \pm 0.01$ & $0.00 \pm 0.01$ & $0.47 \pm 0.12$ & $0.04 \pm 0.01$ & $0.10 \pm 0.02$ & $0.01 \pm 0.005$ \\
\hline 12 & $0.25 \pm 0.14$ & $0.02 \pm 0.01$ & $0.84 \pm 0.12$ & $0.05 \pm 0.01$ & $0.17 \pm 0.03$ & $0.02 \pm 0.004$ \\
\hline 9 & $0.25 \pm 0.12$ & $0.02 \pm 0.01$ & $0.81 \pm 0.14$ & $0.06 \pm 0.01$ & $0.17 \pm 0.03$ & $0.02 \pm 0.003$ \\
\hline 3 & $1.02 \pm 0.17^{\star}$ & $0.06 \pm 0.01^{*}$ & $1.93 \pm 0.36^{\star}$ & $0.10 \pm 0.01^{*}$ & $0.22 \pm 0.03$ & $0.03 \pm 0.002$ \\
\hline 0 & $1.62 \pm 0.57^{\star}$ & $0.09 \pm 0.01^{*}$ & $2.02 \pm 0.29^{\star}$ & $0.13 \pm 0.01^{*}$ & $0.36 \pm 0.03^{\star}$ & $0.03 \pm 0.003$ \\
\hline
\end{tabular}

Data collected from all six animals (mean \pm SEM). ANOVA $(\alpha=0.05)$ with Bonferroni's correction for multiple comparisons. Auto-PEEP, intrinsic positive end-expiratory pressure; MV, controlled mechanical ventilation. *Statistical differences.

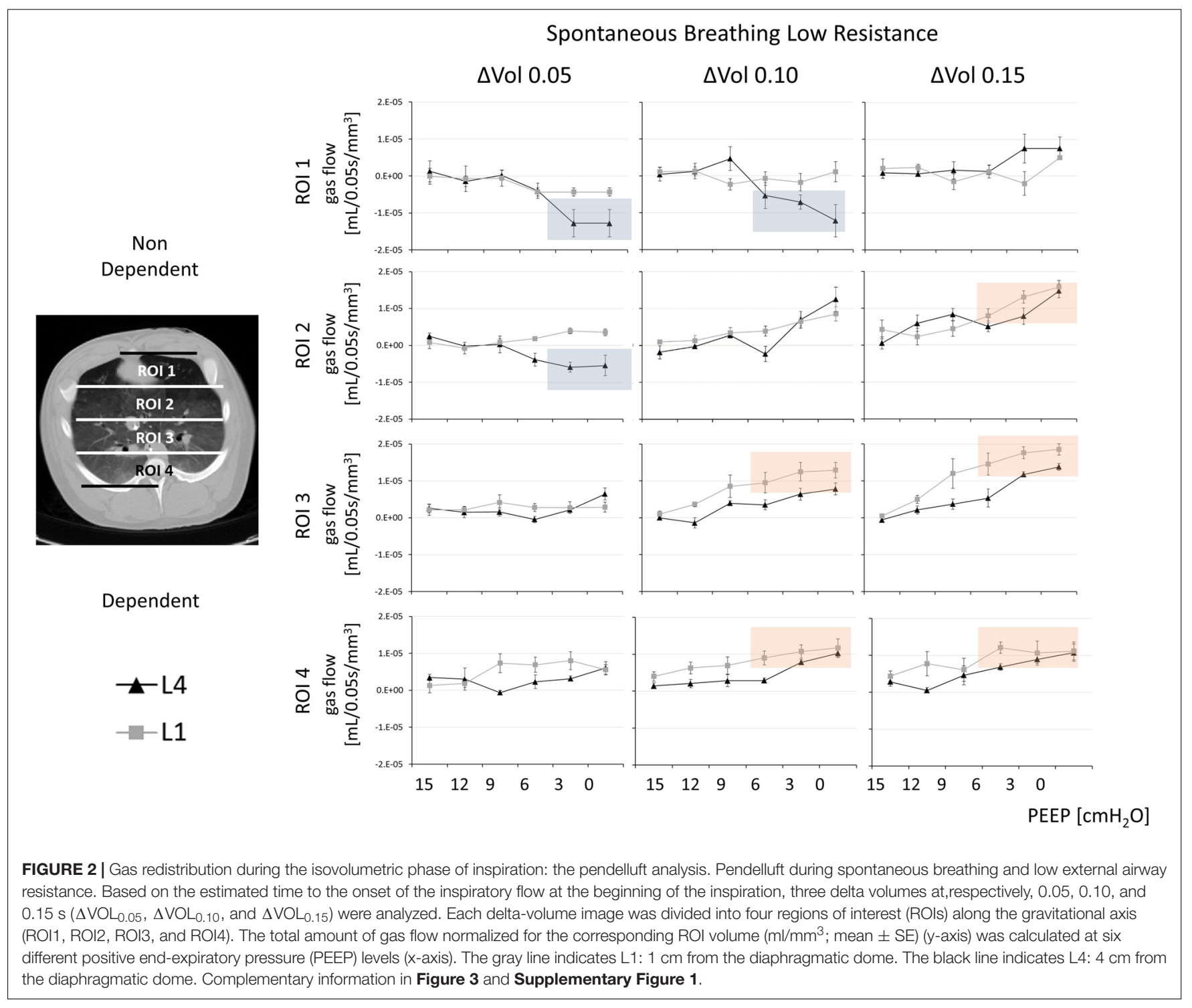

L1) inflation (during $\Delta \mathrm{VOL}_{0.10}$ and $\Delta \mathrm{VOL}_{0.15}$ ) (see Figure 2 and Supplementary Figure 1, blue squares to highlight deflation, red squares to highlight inflation and Supplementary
Tables 1, 2). This intraparenchymal gas redistribution was significantly higher during SB-HighR than during SB-LowR $(p<0.03$ for statistical differences between gas flow during 


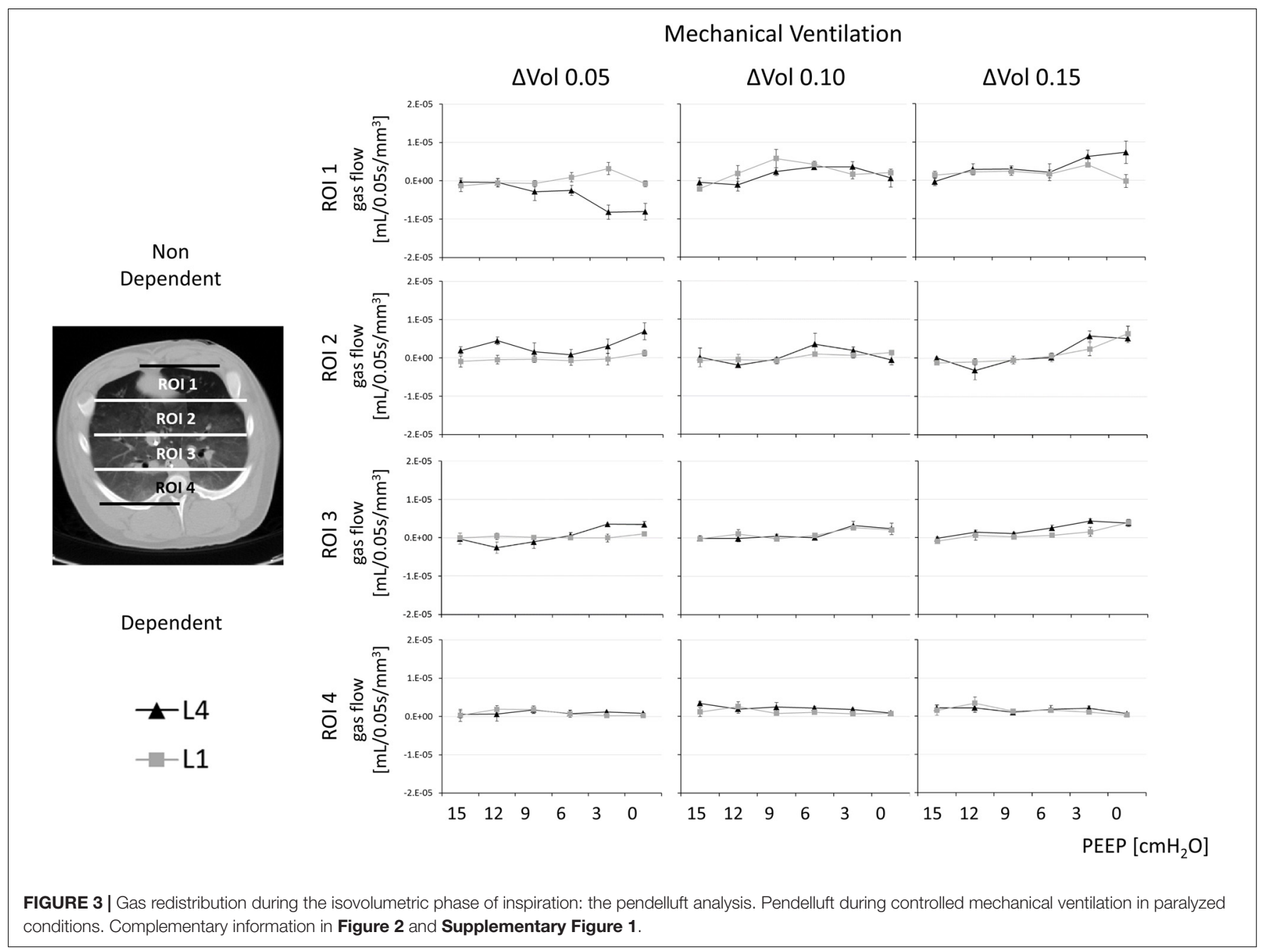

SB-LowR and SB-HighR and PEEP equal or lower than $6 \mathrm{~cm} \mathrm{H}_{2} \mathrm{O}$ for both $\Delta \mathrm{VOL}_{0.05}$ ROI1 and $\Delta \mathrm{VOL}_{0.15}$ ROI4; see Supplementary Table 3). During controlled mechanical ventilation, independently from the selected PEEP level, no pendelluft was observed (see Figure 3).

\section{Intraparenchymal Gas Redistribution During Ongoing Inspiratory Flow}

Sequences of $\triangle$ VOLs allowed a qualitative analysis of the intraparenchymal gas redistribution throughout inspiration. A visual inspection of sequences of the $\triangle$ VOLs showed continuous gas redistribution throughout the entire inspiration; multiple temporary and local transients of gas redistribution continuously occurred independently from the ventilatory conditions (see Figure 4).

The quadtree decomposition algorithm identified two gasredistribution patterns during ongoing inspiratory flow at the airway opening: (1) few and large areas of gas redistribution, referred to as gas displacing (AreaSq equal to or larger than $2 \mathrm{~cm}^{2}$ ); (2) several and small areas of gas redistribution, referred to as gas scattering (AreaSq smaller than $2 \mathrm{~cm}^{2}$ ).
During spontaneous breathing and low external resistances (SB-LowR), high PEEP levels (equal to or higher than $6 \mathrm{~cm}$ $\mathrm{H}_{2} \mathrm{O}$ for $\mathrm{L} 4$ and equal to or higher than $3 \mathrm{~cm} \mathrm{H}_{2} \mathrm{O}$ for $\mathrm{L} 1$ ) were associated with small value of AreaSq (gas scattering) (see Figure 5 and Supplementary Table 4). A higher external resistance (SB-HighR) applied to the airway caused a significant increase in AreaSq (gas displacing) for all PEEP levels equal to or lower than $12 \mathrm{~cm} \mathrm{H} \mathrm{H}_{2} \mathrm{O}$ (see Supplementary Table 5). This was seen at both one (L1) and four (L4) $\mathrm{cm}$ cranially to the diaphragmatic dome.

Controlled mechanical ventilation caused a considerable increase in AreaSq (gas displacing) (see Figure 5 and see Supplementary Table 5). At PEEP of $15 \mathrm{~cm} \mathrm{H}_{2} \mathrm{O}$, the AreaSq value during controlled mechanical ventilation was not significantly different from that during spontaneous breathing. For lower PEEP levels, the AreaSq value was significantly larger (toward the gas-displacing limit) during controlled mechanical ventilation than during spontaneous breathing, independently from the distance from the diaphragm (L1 and L4) (see Figure 5 and see Supplementary Table 6).

The changes toward gas displacing characterizing low PEEP, already described during spontaneous breathing, were also 

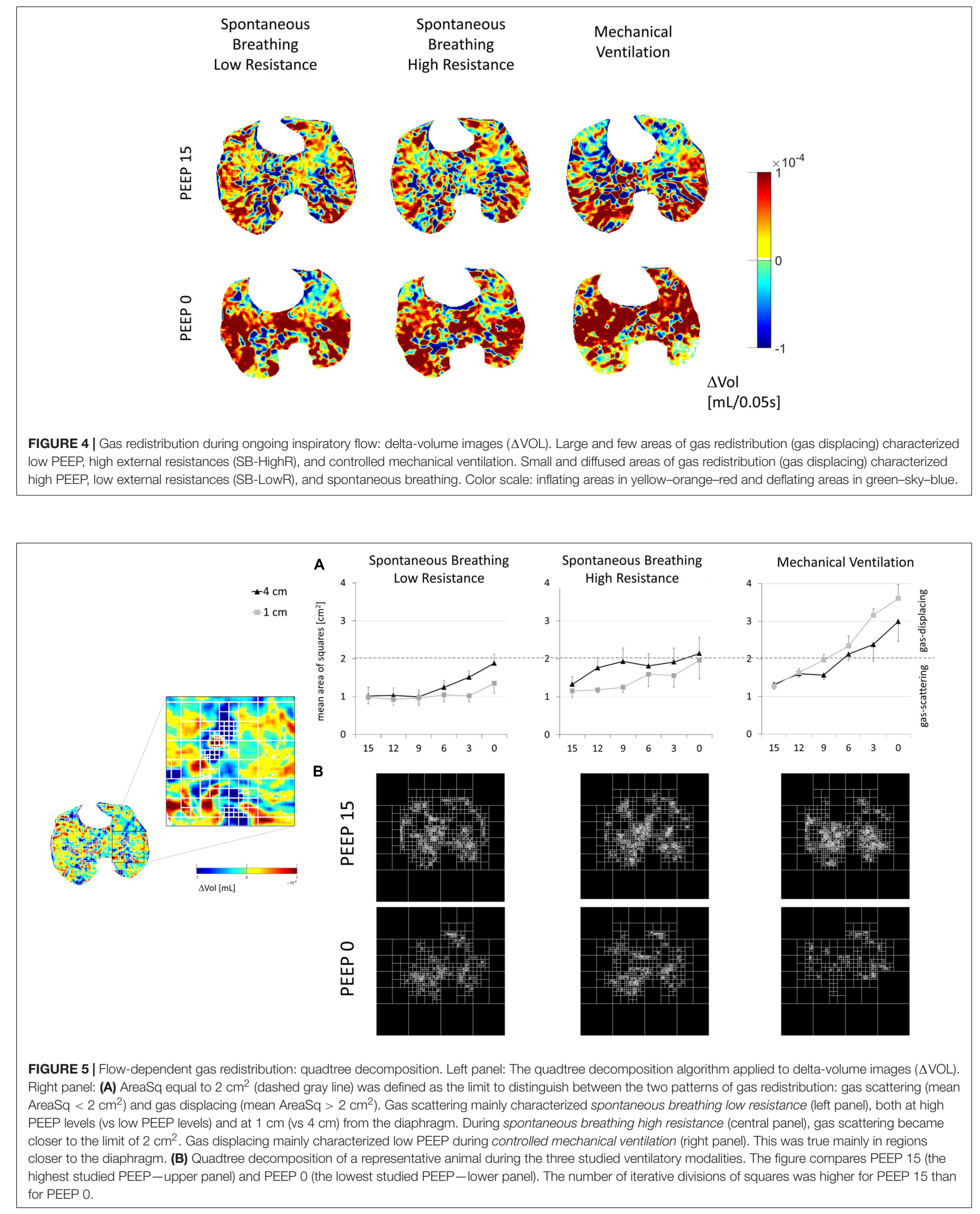
maintained during controlled mechanical ventilation. However, gas displacing was much more emphasized during controlled mechanical ventilation than during spontaneous breathing (see Figure 5 and Supplementary Tables 4, 5).

During spontaneous breathing, the lung regions close to the diaphragm (L1) showed more gas scattering (small AreaSq) than the cranial regions (L4). Conversely, controlled mechanical ventilation caused larger gas displacing (large AreaSq) in caudal regions proximal to the diaphragm (L1) than in more cranial ones (see Figure 5 and Supplementary Table 6).

\section{DISCUSSION}

In the current study, we analyzed temporal and spatial patterns of intraparenchymal gas redistribution in an animal model of mild ARDS. We showed that ventilation of heterogeneous lungs caused transient micromechanical events that trigger the onset of multifold, temporary intraparenchymal gas-redistribution phenomena. This continuously occurred throughout the whole inspiration, during both spontaneous breathing and controlled mechanical ventilation. Therefore, the highly debated pendelluft (Yoshida et al., 2013, 2016a) was only one of the possible inspiratory gas-redistribution phenomena observed. The intraparenchymal gas redistribution occurring during inspiratory flow exhibited two different patterns: (1) gas displacing (i.e., few large areas of gas redistribution) or (2) gas scattering (i.e., several small areas of gas redistribution) (see Figure 4).

Inspiratory efforts during spontaneous breathing, lung volume, owing to different PEEP values, and external airway resistance consistently influenced intraparenchymal gas redistribution. During spontaneous breathing, but not during controlled mechanical ventilation, low lung volume (low PEEP) and high external airway resistances promoted pendelluft, amplifying the initial inspiratory efforts (see Figures 2, 3). Throughout the subsequent flow-dependent inspiratory phase, low PEEP and high external resistances changed the gasredistribution pattern toward larger areas (gas displacing) of gas redistribution (see Figure 5). Although free from pendelluft, controlled mechanical ventilation was characterized by multifold transients of gas redistribution in the second phase of the inspiration. The loss of diaphragmatic tone can justify the onset of larger areas of intraparenchymal gas redistribution during controlled mechanical ventilation.

\section{Intraparenchymal Gas Redistribution Before the Onset of Inspiratory Flow (Pendelluft)}

Pendelluft was found only during spontaneous breathing (see Figures 2, 3). The inspiratory effort characterizing the early phase of the inspiration was defined by measuring the pressure signal during the time needed to generate a flow at airway opening by overcoming the auto-PEEP (see Table 2). The absence of airway flow at the beginning of the inspiratory efforts was mainly attributable to the auto-PEEP. The negative transpulmonary pressure, generated to counteract the auto-PEEP, induces intrapulmonary gradients of pressure and promotes a redistribution of gas within the lung, independently from the inspiratory flow at the airway opening (Mead et al., 1970).

In our study, the magnitude of pendelluft and its topographical distribution were influenced by PEEP and external airway resistances. Low PEEP (lower than $9 \mathrm{~cm} \mathrm{H}_{2} \mathrm{O}$ ) as well as high external airway resistances promoted the onset of pendelluft from nondependent (ROI1) to dependent (ROI4) and from cranial (L4) to caudal (L1) regions of the lung (see Figures 2, 3). Although the effects of higher PEEP in reducing pendelluft was already known before (Yoshida et al., 2016b; Morais et al., 2018), we described this event in a three-dimensional manner, showing a cranial-to-caudal gas redistribution and emphasizing the effects of auto-PEEP and inspiratory efforts, as well as of high external resistances on the onset of pendelluft. Promoting auto-PEEP and more intense inspiratory efforts, high external resistances impeded flow at the airway opening. This consequently facilitated gas redistribution within the lung parenchyma. The increase in external resistance is a complication commonly occurring in intubated and mechanically ventilated critically ill patients. High external resistances are indeed easily promoted by small-sized endotracheal tubes and by tracheal secretions (El-Khatib et al., 2008).

During controlled mechanical ventilation, pendelluft did not occur (see Figure 3). This was presumably attributable to the fact that during the initial isovolumetric phase of the inspiration, there was neither active inspiratory efforts nor enough auto-PEEP. Therefore, no forces were acting on heterogeneous portions of the lung to initiate intraparenchymal gas redistribution (pendelluft).

The main advantage of our technique, based on dynamic computed tomography, is the possibility of defining pendelluft by high spatial resolution. Several studies have shown that the pendelluft can be easily diagnosed at bedside by electrical impedance tomography (EIT) (Karagiannidis et al., 2018; Rossi et al., 2019; Santini et al., 2019; Sang et al., 2020). Although presenting a lower spatial resolution than CT, the EIT is a noninvasive, easily repeatable lung-imaging technique preventing exposure to ionizing radiation.

\section{Intraparenchymal Gas Redistribution During Ongoing Inspiratory Flow}

Although the total amount of gas redistribution was comparable between controlled mechanical ventilation and spontaneous breathing (see Figure 4), larger areas of gas redistribution (gas displacing) characterized controlled mechanical ventilation, while smaller and numerous areas (gas scattering) characterized spontaneous breathing. As for the onset of pendelluft, gas redistribution was significantly influenced by the tested ventilatory conditions. PEEP levels higher than $6 \mathrm{~cm} \mathrm{H}_{2} \mathrm{O}$ and low external airway resistance facilitated the transition toward gas scattering (see Figure 5). In this late phase of the inspiration, the inspiratory effort was defined by the esophageal pressure and the EAdi peak (see Table 1). High inspiratory effort characterized low PEEP levels. The ratio 
between the tidal volume and the corresponding EAdi peak during spontaneous breathing, expression of neuroventilatory efficiency, was increased at PEEP levels between 6 and $12 \mathrm{~cm} \mathrm{H}_{2} \mathrm{O}$, owing to an optimal force-length relationship of the diaphragmatic muscular fibers (Evans and Hill, 1914; see Table 1).

Widespread and highly intermingled transients of gas redistribution resulted in a more extended interface and lower gradients of pressure between temporarily inflating and deflating lung regions. This can act as a lung stabilizer and can explain the reduction in atelectasis and the improved oxygenation observed in optimized spontaneous breathing (Putensen et al., 2001; Wrigge et al., 2003; Levine et al., 2008). These findings are well supported by the theory of emergent phenomena (Andersen, 1972; Macklem, 2008) for which continuous and multiple interactions between temporarily inflating and deflating microareas create a macroscopic stability of the entire lung parenchyma, as previously demonstrated in asthma models (Winkler and Suki, 2011; Winkler et al., 2015). Alveolar interdependence and microinstabilities promote macroscopic lung stability (Faffe and Zin, 2009). Alveoli are interconnected in functional structures. Each single unit benefits from mutual support (Mead et al., 1970). These events are even more pronounced in heterogeneously injured lungs in which anisotropic alveolar mechanics is prevalent (Schiller et al., 2001).

One more difference between spontaneous breathing and controlled mechanical ventilation reported in our study was the inversion of the direction of gas redistribution along the cranialcaudal axis (see Figure 5). During spontaneous breathing, paradiaphragmatic regions of the lung (L1) were characterized by smaller areas of gas redistribution (gas scattering) if compared to more cranial regions of the lung (L4). During controlled mechanical ventilation, the lung close to the diaphragm (L1) showed larger areas of gas redistribution (gas displacing) if compared to more cranial regions (L4). During controlled mechanical ventilation, the loss of diaphragmatic tone and its cranial displacement determined paradiaphragmatic atelectasis and loss of alveolar interdependency (Reber et al., 1998), explaining the presence of a larger area of gas redistribution (gas displacing) in the more caudal region.

\section{LIMITATIONS OF THE STUDY}

The present study has several limitations.

(1) The analysis of gas redistribution reported in the present study is exclusively focused on the inspiratory phase. Further studies are necessary for better characterizing these events over the entire breath.

(2) We studied only continuous positive airway pressure during spontaneous breathing. The choice of continuous positive airway pressure (CPAP) as "ventilatory mode" was due to the will of excluding any interference that a ventilatory support would have had on the spontaneous breathing pattern of the animal. Assisted mechanical ventilation or triggered controlled ventilatory modalities may be characterized by completely different patterns of intraparenchymal gas redistribution.

(3) The current study is based on an animal model of mild ARDS. Swine anatomy substantially differs from human anatomy in terms of diaphragmatic shape, airway displacement, and lung parenchyma. Limitations deriving from anatomical differences should be taken into account when interpreting experimental translational studies. Whether the physiological mechanisms and the pathophysiological implications of these observations could be applied also on patients would require purposely designed clinical studies.

(4) We studied only one specific model of mild ARDS characterized by surfactant depletion. A difference in intraparenchymal gas redistribution among different animal models of lung injury may be hypothesized. Further detailed information about technical aspects of the study has been provided in the Supplementary Material.

\section{CONCLUSION}

Based on a method characterized by higher spatial and temporal resolution, our study described the complexities of inspiratory gas redistribution events. We showed that pendelluft exclusively characterized spontaneous breathing. Suitable PEEP levels and low external resistances, limiting the inspiration efforts, reduced the onset of pendelluft.

Although "free" from pendelluft, controlled mechanical ventilation promoted large intraparenchymal gas redistribution, affecting interdependency among small functional lung units and threatening lung stability.

\section{DATA AVAILABILITY STATEMENT}

The original contributions presented in the study are included in the article/Supplementary Material, further inquiries can be directed to the corresponding author/s.

\section{ETHICS STATEMENT}

The animal study was reviewed and approved by Uppsala regional animal ethics committee (No. C 46_14).

\section{AUTHOR CONTRIBUTIONS}

MP and GP designed and performed the experiments, measured respiratory mechanics, wrote and applied the programs for data analysis, drafted the manuscript, and revised the manuscript for content. GH and AL designed the experiments, analyzed the data, coordinated the activities, drafted the manuscript, and revised the manuscript for content. All authors contributed to the article and approved the submitted version. 


\section{FUNDING}

This study was supported by grants from the Swedish Heart Lung Foundation (no. 20170531), the Swedish Research Council (reg. num. 2018-02438), and the European Society of Intensive Care Medicine (NEXT Grant Award 2017).

\section{ACKNOWLEDGMENTS}

We wish to express our sincere gratitude to Agneta Roneus, Kerstin Ahlgren, Monika Hall, and Anders Nordgren

\section{REFERENCES}

Andersen, P. W. (1972). More is different. Science 177, 393-396. doi: 10.1126/ science.177.4047.393

Brochard, L., Slutsky, A., and Pesenti, A. (2017). Mechanical ventilation to minimize progression of lung injury in acute respiratory failure. Am. J. Respir. Crit. Care Med. 195, 1-16. doi: 10.1164/rccm.201605-1081CP

El-Khatib, M. F., Husari, A., Jamaleddine, G. W., Ayoub, C. M., and BouKhalil, P. (2008). Changes in resistances of endotracheal tubes with reductions in the cross-sectional area. Eur. J. Anaesthesiol. 25, 275-279. doi: 10.1017/ S0265021507003134

Evans, C. L., and Hill, A. V. (1914). The relation of length to tension development and heat production on contraction in muscle. J. Appl. Physiol. 49, 10-16.

Faffe, D. S., and Zin, W. A. (2009). Lung parenchymal mechanics in health and disease. Physiol. Rev. 89, 759-775. doi: 10.1152/physrev.00019.2007

Greenblatt, E. E., Butler, J. P., Venegas, J. G., and Winkler, T. (2014). Pendelluft in the bronchial tree. J. Appl. Physiol. 117, 979-988. doi: 10.1152/japplphysiol. 00466.2014

Harada, K., Nobuo, S., Kisaku, I., Nobumasa, H., Mahito, S., and Kenji, I. (1983). Experimental pendulum air in the flail chest. Jpn. J. Surg. 13, 219-226.

Haren, F. Van, Brochard, L., Bellani, G., Laffey, J., Dres, M., Fan, E., et al. (2019). Spontaneous breathing in early acute respiratory distress syndrome: insights from the large observational study to UNderstand the global impact of severe acute respiratory failure study. Crit. Care Med. 47, 229-238. doi: 10.1097/CCM. 0000000000003519

Karagiannidis, C., Waldmann, A. D., Róka, P. L., Schreiber, T., Strassmann, S., Windisch, W., et al. (2018). Regional expiratory time constants in severe respiratory failure estimated by electrical impedance tomography: a feasibility study. Crit. Care 22, 221. doi: 10.1186/s13054-018-2137-3

Levine, S., Nguyen, T., Taylor, N., Friscia, M., Budak, M., Rothenberg, P., et al. (2008). Rapid disuse atrophy of diaphragm fibers in mechanically ventilated humans. N. Engl. J. Med. 358, 1327-1335. doi: 10.1056/NEJMoa1005372

Macklem, P. T. (2008). Emergent phenomena and the secrets of life. J. Appl. Physiol. 104, 1844-1846. doi: 10.1152/japplphysiol.00942.2007

Mead, J., Takishima, T., and Leith, D. (1970). Stress distribution in lungs: a model of pulmonary elasticity. J. Appl. Physiol. 28, 596-608.

Morais, C., Koyama, Y., Yoshida, T., Plens, G., Gomes, S., Lima, C., et al. (2018). High positive end-expiratory pressure renders spontaneous effort non-injurious American journal of respiratory and critical care medicine. Am. J. Respir. Crit. Care Med. 11, 1285-1296. doi: 10.1164/rccm.2017061244OC

Moss, M., Huang, D. T., Brower, R. G., Ferguson, N. D., Ginde, A. A., Gong, M. N., et al. (2019). Early neuromuscular blockade in the acute respiratory distress syndrome. N. Engl. J. Med. 380, 1997-2008. doi: 10.1056/NEJMoa1901686

Otis, A., Mckerrow, C., Bartlett, R., Mead, J., Mcilroy, M., Selver-Stone, N., et al. (1956). Mechanical factors in distribution of pulmonary ventilation. J. Appl. Physiol. 8, 427-443.

Papazian, L., Forel, J., Gacouin, A., Penot-Ragon, C., Perrin, G., Loundou, A., et al. (2010). Neuromuscular blockers in early acute respiratory distress syndrome. N. Engl. J. Med. 363, 1107-1116.

Pellegrini, M., Hedenstierna, G., Roneus, A., Segelsjö, M., Larsson, A., and Perchiazzi, G. (2017). The diaphragm acts as a brake during expiration to for their assistance and support during the experiments at the Hedenstierna Laboratory of Uppsala University, Sweden. We thank Monica Segelsjö for her skillful CT handling.

\section{SUPPLEMENTARY MATERIAL}

The Supplementary Material for this article can be found online at: https://www.frontiersin.org/articles/10.3389/fphys. 2020.618640/full\#supplementary-material

prevent lung collapse. Am. J. Respir. Crit. Care Med. 195, 1608-1616. doi: 10.1164/rccm.201605-0992OC

Perlman, C. E., Lederer, D. J., and Bhattacharya, J. (2010). Micromechanics of alveolar edema. Am. J. Respir. Cell Mol. Biol. 44, 34-39. doi: 10.1165/rcmb.20090005OC

Putensen, C., Zech, S., Wrigge, H., Zinserling, J., Stüber, F., Von Spiegel, T., et al. (2001). Long-term effects of spontaneous breathing during ventilatory support in patients with acute lung injury. Am. J. Respir. Crit. Care Med. 164, 43-49. doi: 10.1164/ajrccm.164.1.2001078

Reber, A., Nylund, U., and Hedenstierna, G. (1998). Position and shape of the diaphragm: implications for atelectasis formation. Anaesthesia 53, 1054-1061.

Rossi, A., Polese, G., Brandi, G., and Conti, G. (1995). Intrinsic positive endexpiratory pressure (PEEPi). Intensive Care Med. 21, 522-536. doi: 10.1007/ BF01706208

Rossi, F. S., Costa, E. L. V., Iope, D. D. M., Pacce, P. H. D., Cestaro, C., Braz, L. Z., et al. (2019). Pendelluft detection using electrical impedance tomography in an infant keep those images in mind. Am. J. Respir. Crit. Care Med. 200, 1427-1429. doi: 10.1164/rccm.201902-0461IM

Sala, H., Fernández, A., Guardia, S., González, A., and Rodenstein, D. (2002). Supramaximal flow in asthmatic patients. Eur. Respir. J. 19, 1003-1007. doi: 10.1183/09031936.02.00652001

Sang, L., Zhao, Z., Yun, P.-J., Frerichs, I., Möller, K., Fu, F., et al. (2020). Qualitative and quantitative assessment of pendelluft: a simple method based on electrical impedance tomography. Ann. Transl. Med. 8, 1216-1216. doi: 10.21037/atm20-4182

Santini, A., Mauri, T., Dalla Corte, F., Spinelli, E., and Pesenti, A. (2019). Effects of inspiratory flow on lung stress, pendelluft, and ventilation heterogeneity in ARDS: a physiological study. Crit. Care 23, 369. doi: 10.1186/s13054-0192641-0

Schepens, T., Dres, M., Heunks, L., and Goligher, E. C. (2019). Diaphragmprotective mechanical ventilation. Curr. Opin. Crit. Care 25, 77-85. doi: 10. 1097/MCC.0000000000000578

Schiller, H. J., McCann, U. G., Carney, D. E., Gatto, L. A., Steinberg, J. M., and Nieman, G. F. (2001). Altered alveolar mechanics in the acutely injured lung. Crit. Care Med. 29, 1049-1055.

Ultman, J. S., Shaw, R. G., Fabiano, D. C., and Cooke, K. A. (1988). Pendelluft and mixing in a single bifurcation lung model during high-frequency oscillation. J. Appl. Physiol. 65, 146-155.

Vyshedskiy, A., and Murphy, R. (2012). Pendelluft in chronic obstructive lung disease measured with lung sounds. Pulm. Med. 2012, 1-6. doi: 10.1155/2012/ 139395

Winkler, T., and Suki, B. (2011). Emergent structure-function relations in emphysema and asthma. Crit. Rev. Biomed. Eng. 39, 263-280. doi: 10.1002/nbm. 3066. Non-invasive

Winkler, T., Venegas, J. G., and Harris, R. S. (2015). Mathematical modeling of ventilation defects in asthma. Drug Discov. Today Dis. Model. 15, 3-8. doi: 10.1016/j.ddmod.2014.02.008

Wrigge, H., Zinserling, J., Neumann, P., Defosse, J., Magnusson, A., Putensen, C., et al. (2003). Spontaneous breathing improves lung aeration in oleic acidinduced lung injury. Anesthesiology 99, 376-384.

Yoshida, T., Fujino, Y., Amato, M., and Kavanagh, B. (2016a). Fifty years of research in ARDS. Spontaneous breathing during 
mechanical ventilation - risks, mechanisms \& management. Am. J. Respir. Crit. Care Med. 27, 985-992. doi: 10.1164/rccm.201604-07 $48 \mathrm{CP}$

Yoshida, T., Roldan, R., Beraldo, M. A., Torsani, V., Gomes, S., De Santis, R. R., et al. (2016b). Spontaneous effort during mechanical ventilation: maximal injury with less positive end-expiratory pressure. Crit. Care Med. 44, 1-11. doi: 10.1097/CCM.0000000000001649

Yoshida, T., Torsani, V., Gomes, S., De Santis, R. R., Beraldo, M. A., Costa, E. L. V., et al. (2013). Spontaneous effort causes occult pendelluft during mechanical ventilation. Am. J. Respir. Crit. Care Med. 188, 1420-1427. doi: 10.1164/rccm. 201303-0539OC
Conflict of Interest: The authors declare that the research was conducted in the absence of any commercial or financial relationships that could be construed as a potential conflict of interest.

Copyright $\odot 2021$ Pellegrini, Hedenstierna, Larsson and Perchiazzi. This is an openaccess article distributed under the terms of the Creative Commons Attribution License (CC BY). The use, distribution or reproduction in other forums is permitted, provided the original author $(s)$ and the copyright owner(s) are credited and that the original publication in this journal is cited, in accordance with accepted academic practice. No use, distribution or reproduction is permitted which does not comply with these terms. 